\title{
Diseño de juegos para el abordaje de lugares geométricos en la formación inicial de profesores de primaria
}

Datos de contacto:
Celina Milani
Instituto Superior Particular
Incorporado “San José”
Adoratrices № 9017
celimilani@gmail.com
Sara Scaglia
Universidad Nacional del Litoral
scaglia@fhuc.unl.edu.ar
Fabiana Kiener
Universidad Nacional del Litoral
fkiener@gmail.com

Datos de contacto:

Celina Milani

Instituto Superior Particular

Incorporado "San José"

Adoratrices № 9017

Sara Scaglia

Universidad Nacional del Litoral

Fabiana Kiener

fkiener@gmail.com

\author{
Celina MILANI \\ Sara SCAGLIA \\ Fabiana KIENER
}

\begin{abstract}
RESUMEN
Durante la formación inicial del profesor de educación primaria, se debe proporcionar la oportunidad para que los estudiantes desarrollen conocimientos que les permitan intervenir de modo efectivo y pertinente en la formación matemática de los niños. Con ese propósito, se lleva a cabo un estudio de caso con un grupo de futuros profesores, con el objetivo de promover una reflexión didáctica y matemática sobre el diseño de actividades lúdicas que posibiliten el surgimiento de lugares geométricos.

Los resultados mostraron que si bien las situaciones permitieron reflexionar acerca de los elementos que determinan dos lugares geométricos (circunferencia y bisectriz), no cumplieron los principios básicos a tener en cuenta durante la estructuración y aplicación de actividades lúdicas. Por esa razón, se propone dedicar espacios para abordar la noción de juego didáctico y sus características durante la formación inicial de docentes de primaria.
\end{abstract}

PALABRAS CLAVE: Diseño de actividades geométricas; Juegos didácticos; Circunferencia; Bisectriz; Formación docente. 


\title{
Game design for the approach of loci in the initial training of primary school teachers
}

\begin{abstract}
During the initial training of the primary education teacher, the opportunity should be provided for students to develop skills that will enable them to intervene effectively and meaningfully in the children's mathematical training. For this purpose, a case study is carried out with a group of future teachers, with the aim of promoting a didactic and mathematical reflection on the design of playful situations that allow the emergence of loci.

Even though the results showed that the situations made possible to reflect on the elements that determine two loci (circumference and bisector), they did not comply with the basic principles to be taken into account during the structuring and implementation of playful activities. For this reason, it is proposed to dedicate spaces to address the notion of didactic play and its characteristics during the initial training of primary teachers.
\end{abstract}

KEYWORDS: Geometric activities design; Didactic games; Circumference; Bisector; Teacher training.

\section{Introducción}

En este artículo se presentan los resultados de una investigación en torno a la problemática de la formación matemática y didáctica de futuros docentes del nivel primario en Argentina.

Se asumen principios básicos de la Teoría Antropológica de lo Didáctico (TAD) (Chevallard, 2013; Bosch \& Gascón, 2009) para justificar las tareas propuestas. Desde esta perspectiva, se reconoce que la formación matemático-didáctica de profesores que se desempeñan en la escolaridad obligatoria constituye una problemática de especial interés para la investigación didáctica (Bosch \& Gascón, 2009; Sierra et al., 2012; Ruiz et al., 2014). El problema general de esta formación se enuncia en los siguientes términos:

¿Cuál es el equipamiento praxeológico necesario (o por lo menos útil) para que los profesores puedan intervenir de manera efectiva y pertinente en la formación matemática de los 
estudiantes (de una determinada etapa educativa) y qué se puede hacer para ayudar a que los profesores dispongan de él? (Ruiz et al., 2014, p.323).

El término praxeológico alude a una estructura posible de unidad y conocimiento (praxeologia), compuesta por la praxis o el saber hacer, y el logos, que refiere a la justificación de la práctica, es decir, "una descripción, explicación o racionalidad mínima sobre lo que se hace, el cómo se hace y el porqué se hace lo que se hace" (Bosch \& Gascón, 2009, p. 92).

Asimismo, en la TAD se asume que:

cualquier proceso de formación toma sentido a partir del estudio de un conjunto de cuestiones problemáticas al que los estudiantes deben aportar elementos de respuesta. La dialéctica entre el planteamiento de cuestiones problemáticas y la construcción de elementos de respuesta constituye así la razón que fundamenta el proceso de formación (Sierra et al., 2012, p.234)

La formación inicial de docentes para el nivel primario en Argentina se desarrolla en instituciones de nivel terciario (Institutos de Formación Docente) que dependen de las jurisdicciones provinciales. Cada provincia cuenta con un Diseño Curricular propio avalado por las autoridades nacionales para que el título tenga validez en todo el territorio nacional. La investigación se lleva a cabo en un Instituto de Formación Docente de la ciudad de Santa Fe (provincia de Santa Fe), durante el cursado de la asignatura Matemática y su Didáctica II del plan de estudios.

En el Diseño Curricular para la Formación de Profesores de Educación Primaria de la Provincia de Santa Fe, se afirma que "La formación de los/as futuros/as maestros/as tiene como uno de sus propósitos asociar lo más estrechamente posible una formación en matemática, con una reflexión sobre la enseñanza de esta disciplina en la escuela primaria" (Gobierno de Santa Fe. Ministerio de Educación, 2009, p.64). Asimismo, en un estudio encomendado por el Instituto Nacional de Formación Docente, Sadovsky (2010, p.11) reconoce la necesidad de promover en la formación de maestros "una indagación crítica -una re-visión- de los contenidos matemáticos de la escuela primaria [que posibilite] encontrar nuevas relaciones y sentidos".

Los conocimientos geométricos ocupan un lugar relevante en el currículum de la educación primaria (Bernabeu et al., 2018), dado que:

la geometría forma parte de nuestro lenguaje cotidiano, tiene importantes aplicaciones en problemas de la vida real, se usa en 
todas las ramas de la matemática, sirve de base para comprender conceptos de matemática avanzada y de otras ciencias, es un medio para desarrollar la percepción espacial y la visualización (Rodríguez, 2015, p. 12).

En consonancia con estas consideraciones, las situaciones propuestas en la investigación apuntan a recuperar con los futuros docentes saberes geométricos que forman parte del currículum del nivel primario y a promover una reflexión didáctica acerca del diseño de actividades lúdicas para abordar dichos saberes con niños del nivel mencionado.

Diversos estudios exploran la potencialidad de los juegos para el aprendizaje de la geometría en primaria (Bernabeu et al., 2018; Bravo et al., 2013; Franco \& Simeoli, 2019). Dos estudios realizados en Argentina discuten sobre el diseño de juegos por parte de docentes y futuros docentes para la clase de matemática. Collado et al. (2011) investigan "el cambio de concepciones de estudiantes [futuros docentes] y docentes sobre la enseñanza y el aprendizaje de la matemática a partir de su participación en propuestas didácticas que incluyeron el juego" (p. 3). Los autores implementan talleres en los que se proponen juegos motrices y actividades expresivas, que fueron reconocidos por los participantes como experiencias relevantes para comprender otro modo de abordar contenidos matemáticos. Brinnitzer, Collado et al. (2018), por su parte, diseñan un dispositivo que incluye la planificación, implementación y evaluación de secuencias de enseñanza por parte de docentes de primaria en ejercicio.

Esta investigación se enfoca en el diseño, implementación y análisis, por parte de futuros docentes del nivel primario, de situaciones lúdicas para el abordaje de nociones geométricas. Los sujetos de estudio (futuros maestros) deben diseñar juegos para implementar con niños del nivel primario, que habiliten el surgimiento de circunferencia y bisectriz como lugares geométricos (habiendo participado previamente de una situación lúdica para el abordaje del lugar geométrico mediatriz de un segmento). Con el fin de analizar las situaciones diseñadas, éstas se ponen en práctica en el mismo grupo de futuros profesores, quienes posteriormente reflexionan acerca de las potencialidades y limitaciones de las mismas, desde los puntos de vista matemático y didáctico.

La decisión de trabajar con lugares geométricos responde a distintas razones. Por un lado, porque se incluyen en las propuestas curriculares para la educación primaria y para la formación inicial de docentes de ese nivel. Por otro, porque mediatriz, circunferencia y bisectriz constituyen conocimientos básicos para el estudio de innumerables figuras y propiedades geométricas. Como sostienen 
Bernabeu et al. (2017), el reconocimiento de atributos de las figuras y de la relación entre éstos desempeña un papel relevante en la clasificación de las figuras geométricas, actividad de especial interés durante el estudio de la geometría en la escolaridad obligatoria (Gascón, 2004).

En el siguiente apartado se describen los referentes teóricos en los que se enmarca la investigación. Luego se presenta el enfoque metodológico, con una breve descripción del contexto del estudio y de las consignas propuestas para la recolección de datos. Posteriormente se describen los resultados, mediante una breve caracterización de las actividades diseñadas por los futuros docentes. Para finalizar, se discuten los resultados y se presentan las conclusiones.

\section{Referentes teóricos}

En el marco de la TAD, Chevallard (2013) afirma que los saberes matemáticos son obras que tienen una o varias razones de ser, que motivaron su creación y su empleo. Este autor sostiene una mirada crítica sobre la tendencia a ocultar en la enseñanza de la disciplina las razones de ser de los saberes, que suelen ser valorados por sí mismos. Los saberes "se convierten, pues, en monumentos que uno visita, que uno reverencia y frente a los cuales conviene inclinarse sin siquiera intentar conocer las razones de ser que antaño le dieron vida" (p. 25). Para Chevallard (2013, p.25) este paradigma didáctico de la visita de monumentos "tiende a dotar de poco sentido las obras así visitadas", dado que las preguntas referidas a la utilidad de estas obras, a las razones de su estudio, no son respondidas.

Bosch et al. (2006) afirman que una cuestión matemática se estudia con sentido en la escuela, si se cumplen los siguientes postulados:

- Legitimidad cultural o social: debe surgir de cuestiones que la sociedad propone que se estudien en la escuela.

- Legitimidad matemática: debe aparecer en determinadas situaciones ubicadas en la raíz central de las matemáticas.

- Legitimidad funcional: debe estar relacionada con otras cuestiones que se estudian en la escuela (de las matemáticas o de otras disciplinas).

Si determinada cuestión no cumple con los postulados anteriores, se trata de "una cuestión encerrada en sí misma (o muerta) porque se ignora el por qué y el para qué de su estudio escolar" (Gascón, 2004, p.41).

Con el fin de dotar de sentido a los saberes matemáticos, en la TAD se postula la necesidad de identificar cuestiones generatrices, 
preguntas medulares, para las cuales los saberes matemáticos son necesarios para dar respuesta. En particular, para los saberes geométricos, Gascón (2004) propone los siguientes interrogantes: “¿Cuáles son los elementos que determinan un tipo determinado de figuras?, ¿Existen diferentes sistemas de elementos que determinan el mismo tipo de figuras?, ¿Cuál de ellos es el más adecuado para utilizarlo en determinada situación de construcción?” (Gascón, 2004, p.42). Estas preguntas son consideradas en el diseño de las consignas que se proponen a los futuros maestros.

Otra cuestión central en las consignas propuestas es la inclusión de la actividad lúdica. Ortiz (2014) concibe al juego didáctico como una técnica participativa de enseñanza, que "no sólo propicia [en los estudiantes] la asimilación y construcción de conocimientos y el desarrollo de habilidades, sino que además contribuye al logro de la motivación por las actividades docentes" (p.218). Este autor propone principios básicos a tener en cuenta para estructurar y aplicar actividades lúdicas que promuevan el aprendizaje de determinados contenidos específicos en las diferentes asignaturas, a saber:

- Participación: se considera el principio básico de la actividad lúdica. Negar al niño la posibilidad de participar supone limitar su independencia, promover "la aceptación de valores ajenos, y en el plano didáctico implica un modelo verbalista, enciclopedista y reproductivo" (p.221).

- Dinamismo: el juego implica movimiento, desarrollo e interacción activa en la dinámica del proceso pedagógico.

- Entretenimiento: este principio refuerza especialmente el interés y la actividad cognitiva de los jugadores. El juego "no admite el aburrimiento, las repeticiones, ni las impresiones comunes y habituales" (p.222).

- Desempeño de roles: se basa en la modelación lúdica de la actividad del niño. Refleja fenómenos relevantes para el desarrollo del juego como la imitación y la improvisación.

- Competencia: "El valor didáctico de este [principio] es evidente: sin competencia no hay juego, ya que ésta incita a la actividad independiente, dinámica, y moviliza todo el potencial físico e intelectual del niño" (p.222). Para Ortiz (2014), este principio adquiere una relevancia y valor didáctico de primer orden.

Muñiz-Rodríguez et al. (2014) sostienen que el juego favorece la adquisición de los conocimientos matemáticos, dado que promueve el interés y la motivación de los estudiantes. Brinnitzer et al. (2018) destacan la importancia de vincular el juego y los contenidos matemáticos, porque se promueve la oportunidad de aprender en interacción con otros. Las autoras sostienen que esta posibilidad se 
potencia si el docente "recupera las estrategias y los conocimientos en acto construidos por los estudiantes durante la acción, aunque no estén explicitados" (p.311).

\section{Método}

La investigación se enmarca en la modalidad cualitativa interactiva, caracterizada por el empleo de técnicas para recoger datos en escenarios naturales (McMillan \& Schumacher, 2005). Se lleva a cabo un estudio de caso para recolectar información referida a cómo futuros docentes del nivel primario elaboran y analizan situaciones lúdicas para trabajar nociones geométricas en la escuela primaria, teniendo en cuenta las razones de ser de los conceptos involucrados.

El caso analizado es el desempeño de 30 estudiantes (futuros docentes de nivel primario) que cursan la asignatura Matemática y su Didáctica II, de un Instituto de Formación Docente de la ciudad de Santa Fe (Argentina).

Para la recolección de datos se utilizaron grabaciones en audio de las clases, producciones escritas de los estudiantes y registros fotográficos. El análisis de datos consiste en un estudio sistemático de la situación lúdica presentada por cada grupo de estudiantes en torno a la noción geométrica involucrada: si favorece el surgimiento de dicho concepto, si promueve concepciones erróneas y si es posible proponer modificaciones que favorezcan el aprendizaje de dicha noción.

\section{Caracterización de la propuesta}

El plan de estudios para la formación inicial de maestros del nivel primario (Gobierno de Santa Fe. Ministerio de Educación, 2009) se organiza en torno a tres campos de conocimientos (Formación General, Formación en la Práctica Profesional y Formación Específica), y a dos ejes transversales (Itinerario por el Mundo de la Cultura y Taller de Producción Pedagógica). Tiene una duración de cuatro años y la asignatura Matemática y su Didáctica II se propone para el tercer año de estudios, en el campo de la Formación Específica. Este campo "comprende el conjunto de saberes que definen las competencias del ejercicio de la función docente en este nivel" (Gobierno de Santa Fe. Ministerio de Educación, 2009, p.20).

En los Núcleos Interdisciplinarios de Contenidos se menciona la necesidad de que los docentes experimenten, junto a sus estudiantes, propuestas lúdicas (Ministerio de Educación Provincia de Santa Fe, 2016). En el Diseño Curricular para la formación de profesores de 
primaria se proponen espacios curriculares para abordar el juego y la creatividad en la formación de docentes. Desde un punto de vista más específico, vinculado con el conocimiento matemático, uno de los Talleres opcionales propuestos en el marco de las asignaturas Matemática y su Didáctica I y II se denomina "El juego un recurso para enseñar" (Gobierno de Santa Fe. Ministerio de Educación, 2009, p. 106).

Como se indicó anteriormente, las consignas propuestas giran en torno al diseño, implementación y análisis de juegos que permitan abordar lugares geométricos, definidos como conjuntos de puntos de un plano que cumplen determinadas condiciones. De este modo se consideran las orientaciones curriculares y la problemática de la determinación y construcción de las figuras geométricas, cuestión generatriz para la enseñanza de la geometría en la escolaridad obligatoria (Gascón, 2004; Ruiz et al., 2014).

En la Tabla 1 se describen las acciones llevadas a cabo en cada clase de 80 minutos y los trabajos grupales (denominados Tareas) que deben resolver para la próxima clase. Las consignas correspondientes pueden consultarse en Kiener y Milani (2019).

Tabla 1.

Descripción de acciones y consignas de tareas grupales por clase

\begin{tabular}{|l|l|}
\hline CLASE & \multicolumn{1}{c|}{ DESCRIPCIÓN } \\
\hline \multirow{5}{*}{ Clase 1 } & $\begin{array}{l}\text { Implementación de un juego en torno al concepto de mediatriz a cargo } \\
\text { de dos integrantes del equipo de investigadoras (una es la docente del } \\
\text { curso). Participan todos los estudiantes del curso. }\end{array}$ \\
\cline { 2 - 3 } & $\begin{array}{l}\text { Tarea 1: Plantear una actividad con elementos lúdicos que posibilite } \\
\text { introducir la noción matemática asignada (bisectriz, 3 grupos, y } \\
\text { circunferencia, 3 grupos; se designan estos grupos con la sigla GD: } \\
\text { Grupo Diseñador). Mencionar cuáles son los conocimientos previos } \\
\text { que deben tener los estudiantes. Presentar la tarea en forma escrita y } \\
\text { proveerse de los materiales necesarios para su implementación. }\end{array}$ \\
\hline \multirow{5}{*}{ Clase 2 } & $\begin{array}{l}\text { Implementación de las actividades diseñadas por los seis grupos (en } \\
\text { cada una, el GD gestiona su desarrollo y el resto de los estudiantes la } \\
\text { realiza). }\end{array}$ \\
\cline { 2 - 3 } & $\begin{array}{l}\text { Tarea 2: Primer análisis de las actividades diseñadas. } \\
\text { Cada grupo (identificado con la sigla GE: Grupo Evaluador) debe } \\
\text { analizar una actividad que no ha diseñado. Se pide describir el modo } \\
\text { en que emerge el concepto matemático, enunciar las características } \\
\text { del concepto que aparecen, analizar si es adecuado para presentarlo } \\
\text { en 4 }{ }^{\circ} \text { o 5 año de la Escuela Primaria y mencionar qué modificaciones } \\
\text { le harian para optimizar el aprendizaje de la noción involucrada. }\end{array}$ \\
\hline \begin{tabular}{l} 
Puesta en común del primer análisis. \\
\cline { 2 - 3 } Clase 3 3
\end{tabular} & $\begin{array}{l}\text { Tarea 3: Segundo análisis de las actividades diseñadas. } \\
\text { Cada GD debe analizar la actividad que ha diseñado. Se reflexiona } \\
\text { acerca del desempeño de los jugadores (acciones a desarrollar, si }\end{array}$ \\
\hline
\end{tabular}




\begin{tabular}{|l|l|}
\hline & $\begin{array}{l}\text { plantea o no un desafio) y de la producción matemática resultante } \\
\text { (información sobre la noción matemática que se pone en evidencia en } \\
\text { cada actividad, definición que se construye, caracteristica/s } \\
\text { central/es del concepto, si se accede a la construcción de la figura, si } \\
\text { promueve concepciones erróneas en relación con dicha noción). Se } \\
\text { solicita una nueva reflexión acerca de las modificaciones que se } \\
\text { proponen para optimizar el aprendizaje de la noción. }\end{array}$ \\
\hline Clase 4 & $\begin{array}{l}\text { Puesta en común del primer análisis de los grupos faltantes e inicio de } \\
\text { la discusión en torno al segundo análisis. }\end{array}$ \\
\hline Clase 5 & $\begin{array}{l}\text { Finalización de la puesta en común del segundo análisis y cierre de la } \\
\text { experiencia áulica mediante la construcción de las definiciones de } \\
\text { bisectriz y circunferencia. }\end{array}$ \\
\hline
\end{tabular}

\section{Resultados}

Se presenta a continuación una breve descripción de cada una de las actividades diseñadas durante la Tarea 1 (a cargo de los GD). El análisis retoma las consideraciones realizadas por GE y GD luego de la implementación, durante las Tareas 2 y 3, respectivamente. Además, incluye una valoración sobre la pertinencia de la situación para el estudio de los lugares geométricos correspondientes.

\section{Actividades propuestas para abordar la noción de circunferencia}

\section{Actividad del reloj}

Se desarrolla en el patio de la institución y se forman dos equipos de 13 estudiantes cada uno. En cada equipo se debe representar un reloj imaginario. Un estudiante se sitúa en el centro del reloj, sosteniendo una cuerda, y los 12 restantes se ubican (tensando la cuerda) en las posiciones que corresponden a las horas (ver Figura 1).

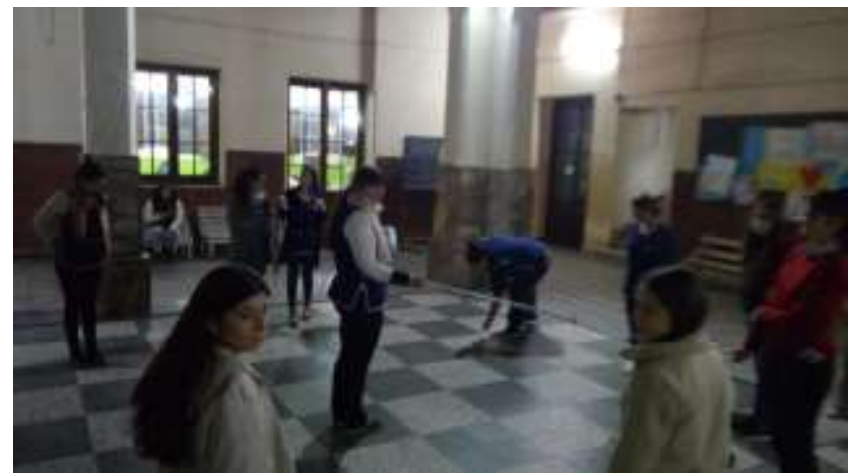

Figura 1. Utilización de la cuerda para representar el reloj.

Una vez situados estos últimos, cada estudiante marca su posición con una cruz en el piso. Luego GD solicita que se unan las cruces con 
una tiza e institucionaliza informando a la clase que la figura que ha quedado dibujada en el piso es una circunferencia.

\section{Análisis de la actividad}

Según los grupos GE y GD, la situación favorece la emergencia del concepto de circunferencia. GD señala que se introducen los elementos radio y centro, que plantea un desafio para los jugadores y que daría lugar a concepciones erróneas si no se hacen explícitas algunas cuestiones (que la cuerda esté tensa y se tome siempre del mismo modo).

Se considera, sin embargo, que la situación no resulta pertinente para la emergencia del concepto de circunferencia, ya que los puntos que indican cada hora del reloj podrian unirse con trazos rectos o curvos (y estos últimos podrian no pertenecer necesariamente a una única circunferencia, como se observa en la foto de la Figura 2).

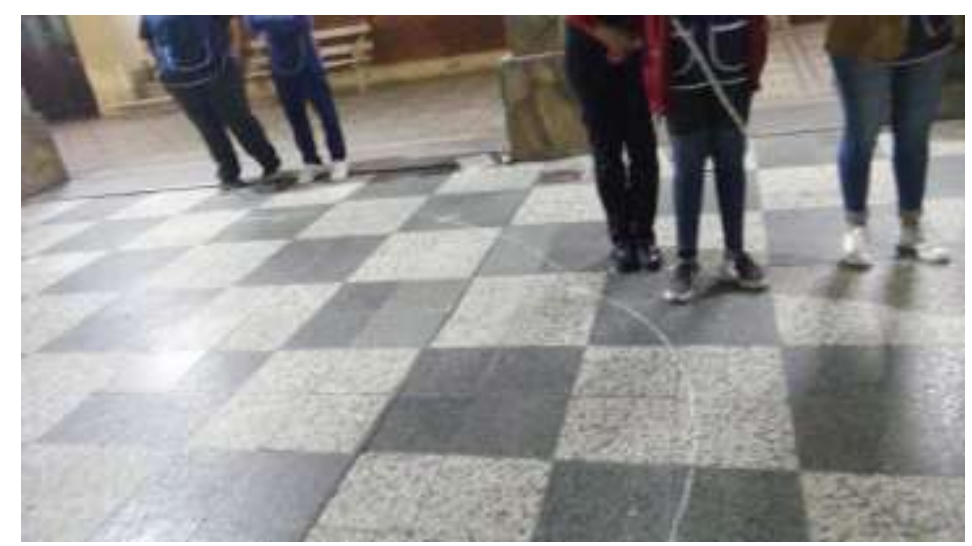

Figura 2. La unión de cruces no determina una circunferencia.

Asimismo, se señala que es posible que el marco de un reloj adopte una forma no circular (por ejemplo, rectangular o poligonal). GD salvó esta limitación haciendo referencia en el escrito a un "reloj convencional", asumiendo que éste es circular.

Para favorecer el aprendizaje de la circunferencia, GD propone "establecer una posición de agarre exacta igual de los extremos de la soga para los doce niños que van a simular los números del reloj", para que los puntos marcados equidisten del centro. Con respecto a esto, se añade la necesidad de que en la consigna se solicite marcar en el piso la trayectoria del extremo libre del segundero, en lugar de unir los puntos marcados con tiza. Esto fue discutido en la puesta en común de la Tarea 3. 


\section{Actividad del auto}

Se desarrolla en el aula. Las integrantes del GD atan un autito de juguete y un fibrón en un extremo de una cuerda. Sujetan el otro extremo a un punto fijo de una plancha de telgopor y luego hacen girar el auto para que se desplace con la cuerda tensa.

El fibrón marca sobre la plancha una trayectoria (ver Figura 3) y GD afirma que se trata de una circunferencia.

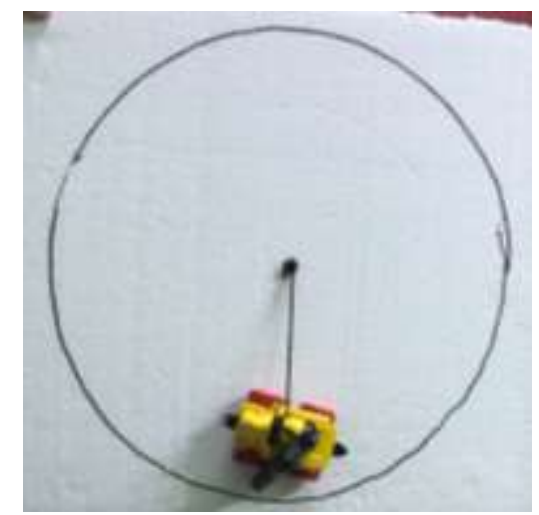

Figura 3. Trayectoria marcada por el fibrón.

Análisis de la actividad

Los estudiantes afirman que emerge el concepto de circunferencia. GE señala que la "circunferencia emerge con la observación del recorrido que realiza el auto de juguete" e indica también los elementos de esta: centro y radio. No se menciona el surgimiento de concepciones erróneas. Tanto GD como GE presentan como limitación el hecho de que la actividad la podría llevar adelante un solo niño y que el resto de la clase tendría una actitud pasiva. Para subsanar esta limitación, GD propone modificar "la base de telgopor por una base mucho más grande en la que todos puedan participar y así lograr que el juego sea más dinámico" y "que al final del juego haya un espacio de debate". GE, por su parte, propone "Formar grupos de 4 o 5 y que cada grupo traiga los materiales y que con preguntas de la docente piensen cómo armar la maqueta".

Se considera que durante la actividad surgen las nociones de centro y radio y que queda de manifiesto que los puntos de la trayectoria equidistan del centro. Si bien en el escrito no explicitan la condición que debe cumplir el hilo (estar tenso), cuando llevan a cabo la acción lo mantienen tenso. Se señala que la ausencia de esta condición podría dar lugar a diferentes trayectorias (no necesariamente que correspondan a una circunferencia). Además, se deberia proponer un contexto que justifique el recorrido de una 
circunferencia (por ejemplo, considerar el recorrido que realiza una figura en un carrusel).

\section{Actividad de la proyección de sombras en la pared}

Se desarrolla en el aula. GD distribuye entre los estudiantes diversos objetos (aros, naranjas, pastel de goma EVA, bandejas de acero inoxidable circulares, entre otros; ver Figuras 4 y 5) y tres linternas.
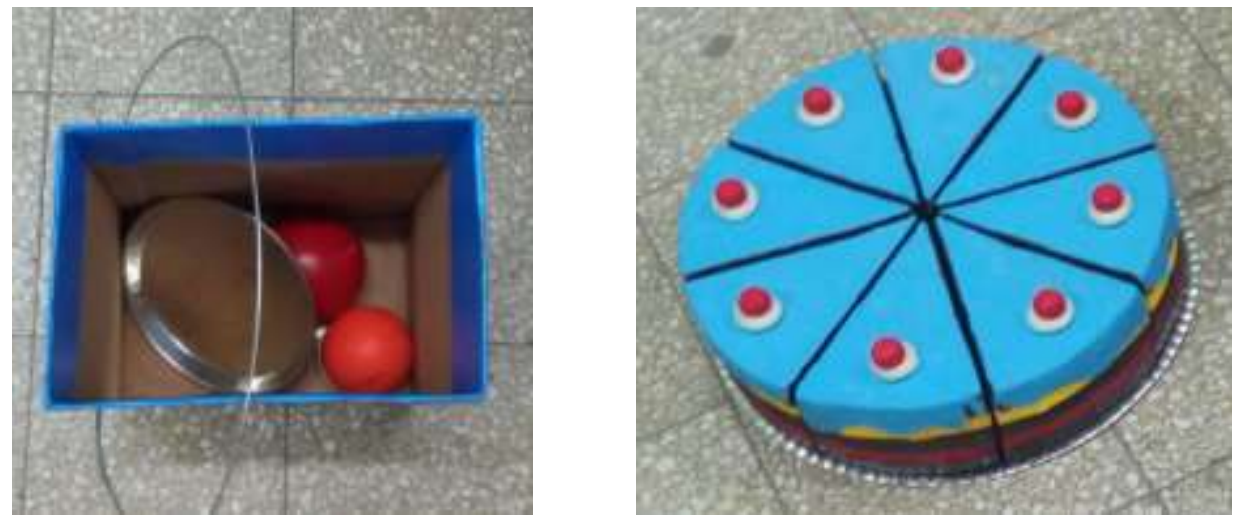

Figuras 4 y 5 . Objetos redondos varios y pastel de goma EVA.

GD solicita que enfoquen con las linternas a estos objetos y observen las sombras que se proyectan sobre las paredes del aula, con la luz apagada (ver Figuras 6 y 7). Luego preguntan qué figuras quedaron determinadas. A partir de las respuestas de los estudiantes, se distinguen las nociones de circunferencia y círculo.
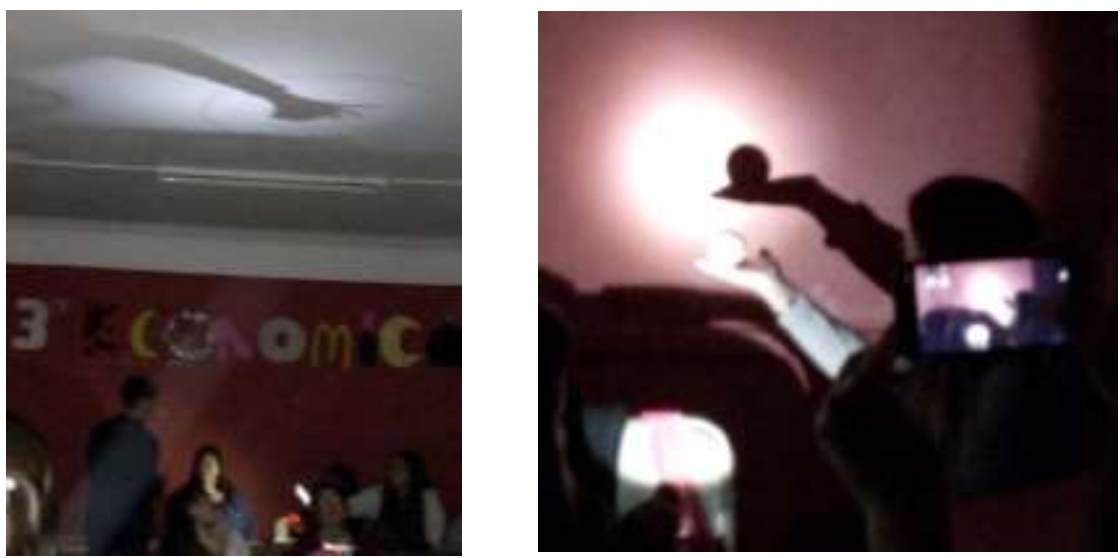

Figuras 6 y 7. Proyección de sombras en el techo y proyección de sobras en la pared. 
Análisis de la actividad

GE afirma que "emerge el concepto trabajado desde conocimientos previos, material concreto [...] el poder manipular los diferentes objetos". GD sostiene que "El juego pone en evidencia la diferencia entre círculo y circunferencia" y que "no ofrece la oportunidad de reflexionar acerca de cuáles y cuántos son los puntos del plano que pertenecen a una circunferencia". Para subsanar estas limitaciones sugieren que el docente proyecte "la sombra del aro o del anillo con el objetivo de establecer el punto medio [es decir, el centro] y el radio de la misma [circunferencia] para que posteriormente los alumnos grafiquen en papel una circunferencia con el compás (conociendo el punto medio y el radio de la misma)". El único cambio sugerido por GE es "pedirles a los alumnos que lleven ellos los materiales".

Se considera que en la actividad, las sombras proyectadas darian lugar a figuras geométricas diversas, además de circunferencias y círculos. Esto requeriría una discusión y reflexión en la clase, para distinguir las diferentes figuras que se presentan y luego detenerse sobre las que el docente desea trabajar especificamente.

\section{Actividades propuestas para abordar la noción de bisectriz}

\section{Actividad del arco}

Se desarrolla en el patio de la institución. GD solicita a los participantes (divididos en tres grupos) seleccionar dos columnas del patio que representarán los postes de un arco de un campo de fútbol. Resultan seleccionados tres pares de columnas. Los participantes de la actividad tienen que elegir un punto del campo desde donde patear un tiro libre. Una vez elegido éste, se pide determinar con cinta o lana sobre el piso los segmentos que lo unen a los dos postes. Posteriormente, se debe marcar con cinta o lana la trayectoria rectilinea que recorrería la pelota pateada por un jugador, para que pase exactamente por el punto medio del arco que está sobre el piso (ver Figura 8). 


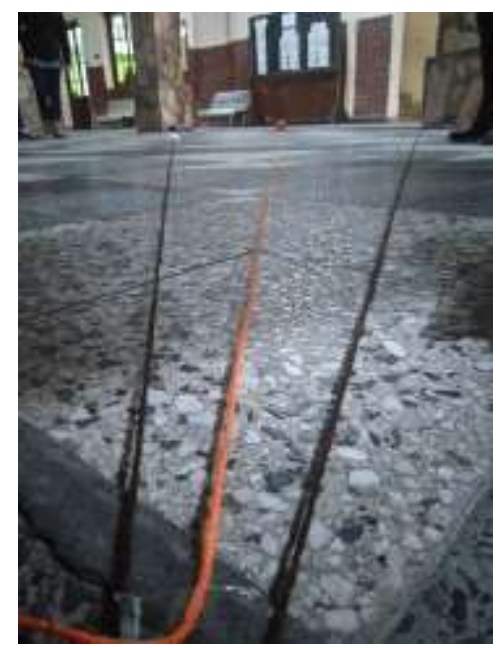

Figura 8. Ángulo marcado con lana en el piso.

Al final, GD afirma que la trayectoria de la pelota forma parte de la bisectriz del ángulo (determinado sobre el plano del piso cuyo vértice es el punto desde donde se ejecuta el tiro libre y cuyos lados pasan por las bases de los postes del arco).

\section{Análisis de la actividad}

GE considera que emerge el concepto de bisectriz "a través de la resolución de un juego que consiste en tomar un arco y con la pelota patear un tiro libre de modo que la pelota ingrese en el medio del arco". Durante la puesta en común de este primer análisis, debido a las preguntas realizadas por la docente para profundizar en las características de las nociones geométricas involucradas, la clase reconoce que la trayectoria de la pelota no coincide con la bisectriz del ángulo, sino con una mediana del triángulo definido entre los tres puntos (tiro libre y bases de los postes).
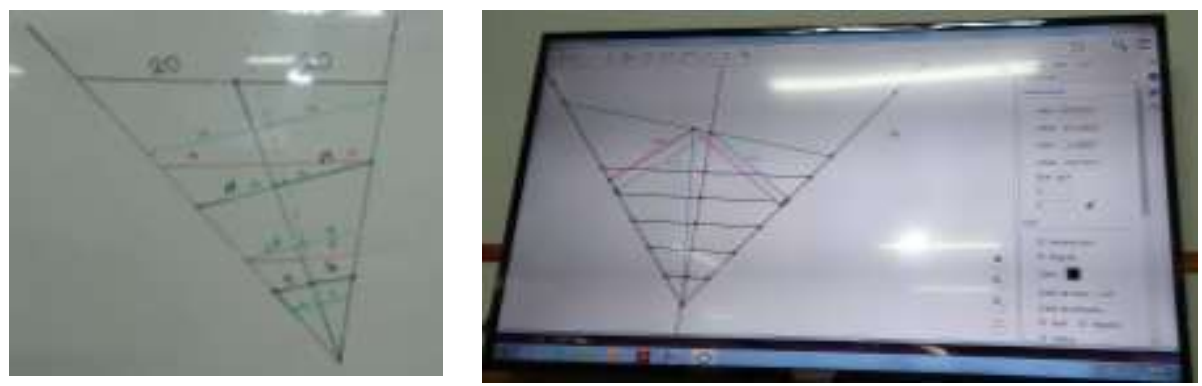

Figuras 9 y 10. Representación en el pizarrón y Representación en GeoGebra. 
Para favorecer esta discusión se realizaron dibujos en el pizarrón (ver Figura 9) y en GeoGebra (ver Figura 10).Como consecuencia de esta reflexión, en la Tarea 3 GD afirma que "el objetivo era que se ponga en evidencia la noción de bisectriz, pero por un mal planteamiento de la actividad, se pone en evidencia la noción de punto medio de un segmento y una línea que divide en dos el ángulo".

Con respecto a las modificaciones propuestas, GE afirma que "deberia estar más especificado el concepto de tiro libre para evitar que todos los grupos lo resuelvan de la misma forma". Esta reflexión tiene que ver con el hecho de que dos de los grupos eligieron, para posicionar la pelota, el punto de tiro penal. Esta posición impidió que se ponga en evidencia el error geométrico mencionado, dado que quedaron determinados triángulos isósceles, en los cuales la mediana del lado desigual coincide con el segmento de bisectriz (ver Figura 11).

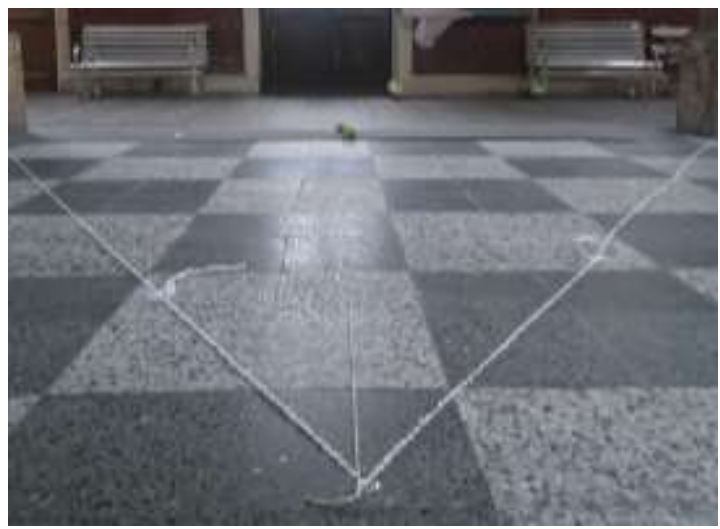

Figura 11. Triángulo isósceles por pelota en posición de tiro penal.

GD, por su parte, propone que "Se podría pedir que, luego de marcar los extremos del arco y la posición de la pelota, como puntos del plano, deberían hacer una marca cada cierta medida en donde se vea el recorrido que haria la pelota. Este recorrido debe equidistar de los lados del ángulo".

Si bien se parte de una concepción errónea del trazado de la bisectriz, la discusión posterior permitió subsanar el error y generar un espacio propicio para trabajar otras nociones, como la distancia de un punto a una recta y el trazado del segmento que la determina, así como la mediana correspondiente a un lado de un triángulo. 
5. Actividad de la búsqueda del tesoro

Se desarrolla en el patio de la institución. GD construye en el piso con cinta adhesiva de papel un triángulo (aparentemente isósceles) que representa el Triángulo de las Bermudas. Una integrante lee un relato en el que se informa el modo de descubrir un tesoro. En el interior del triángulo colocan cuadraditos de cartulina. Piden a las "jugadoras" (tantas jugadoras como papelitos hay) que cada una se pare sobre una cartulina de la que sospeche que representa un barco (las cartulinas que representan barcos tienen dibujada una cruz en el lado oculto), pues éstos señalan el camino a seguir para llegar al tesoro. Luego de que todos los lugares están ocupados, cada uno debe dar vuelta la cartulina sin cambiarla de posición. A continuación se solicita a las jugadoras que unan con segmentos de recta (utilizando cinta adhesiva de papel) los barcos, que están alineadas con uno de los vértices del triángulo (ver figura 12).

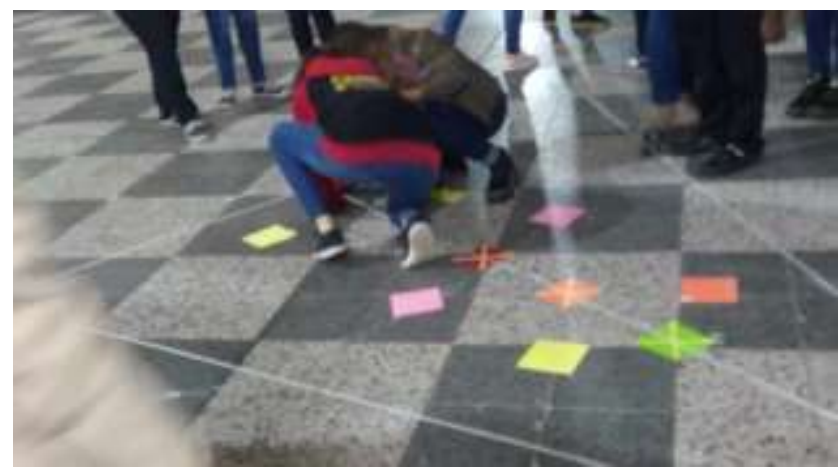

Figura 12. Trazado de segmentos con tiza.

GD afirma que si se prolonga este segmento se verá el camino que conduce a un faro en el que se ha escondido un tesoro. En la prolongación del segmento se ha escondido una bolsa de caramelos, que luego reparten entre los estudiantes del curso.

\section{Análisis de la actividad}

Durante el primer análisis, GE manifiesta que "el concepto no emerge ya que las cruces ya estaban posicionadas formando la bisectriz. El alumno no participa construyendo bisectriz porque ya estaba construida". Además, reconocen que no se ponen de manifiesto las condiciones que deben satisfacer los puntos que pertenecen a la bisectriz y su trazado: "no están demasiado claras las condiciones] ya que trazaron un triángulo en lugar de un ángulo. Lo hicieron de manera contraria y no desde el vértice del ángulo que es donde nace la bisectriz". En el segundo análisis, GD reconoce que la actividad no da lugar a la construcción de la bisectriz, dado que los participantes 
actúan como meros observadores. Además, afirman que puede dar lugar a concepciones erróneas "porque los alumnos podrían haber asimilado el juego con la división en dos partes iguales de un triángulo en lugar que uno de los ángulos del mismo". Con respecto a las modificaciones, sugieren: "Podrian a su vez haber orientado el camino de la recta hacia el lado opuesto del ángulo del triángulo, es decir, hacia su base".

Además de las limitaciones mencionadas por GD y GE, se considera que esta situación no es una actividad lúdica $\mathrm{y}$, como se ha señalado en párrafos anteriores, contiene numerosas imprecisiones. La presentación escrita de la situación es confusa.

\section{Actividad del gimnasta}

Se desarrolla en el patio. GD dibuja en el piso un cuadrado con cinta adhesiva de papel. Indican que representa una plataforma para realizar gimnasia artística. Informan que una gimnasta comienza desde un vértice del cuadrado y se desplaza mediante saltos y giros, avanzando $30 \mathrm{~cm}$ por salto. En cada salto cae en un punto que equidista de los lados del cuadrado (se aclara que se toman los lados que determinan el vértice de donde parte).

Una estudiante adopta el papel de gimnasta y luego se pide a otros estudiantes que, mediante cruces realizadas con cinta adhesiva de papel, señalen los puntos en los que cae en cada salto. Para marcar los puntos, los estudiantes utilizan una cinta métrica (ver Figura 13), pero también se orientan por las baldosas que son cuadradas.
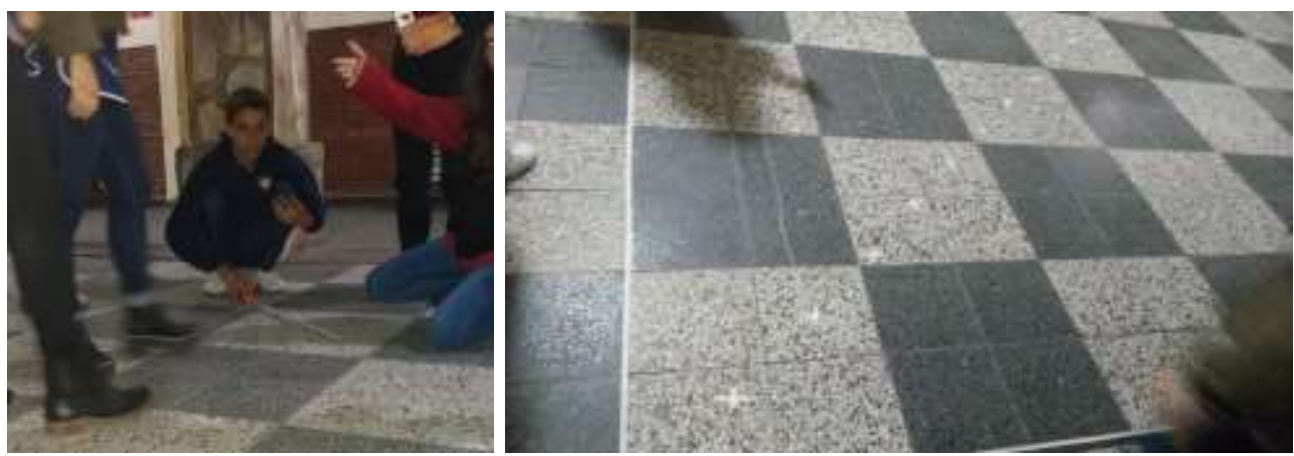

Figuras 13 y 14. Mediciones para determinar los puntos e Hilera de puntos que pertenecen a la bisectriz.

Queda así determinada una hilera de cruces sobre la plataforma (ver Figura 14). GD institucionaliza y afirma que los puntos pertenecen a la bisectriz del ángulo considerado. 
Análisis de la actividad

GE sostiene que "El punto de partida del gimnasta da cuenta de que la bisectriz es una semirrecta que divide en dos partes iguales al ángulo". GD de manera similar afirma que "Mediante el juego se observa de qué manera se traza, cuál es su definición, qué función cumplen los puntos señalados, qué figura geométrica quedó determinada y qué determina la recta trazada en el ángulo establecido".

Con respecto a las modificaciones sugeridas, GE propone "ampliar las longitudes de los saltos del gimnasta, retomar conceptos previos y especificar la cantidad de integrantes". Este grupo considera que resulta inverosímil que la longitud de cada salto sea de $30 \mathrm{~cm}$. Por su parte GD manifiesta que "deberíamos haber brindado más elementos para que logren medir con exactitud las distancias requeridas".

Se considera que el contexto es adecuado y que el enunciado de las pautas es claro y cumple con su objetivo (el surgimiento del concepto de bisectriz, con las condiciones que deben cumplir los puntos que pertenecen a ella). Cabe señalar que, al tratarse de una plataforma cuadrada que se demarca sobre un piso revestido con baldosas cuadradas, se facilita la determinación de los puntos de la bisectriz, dado que coinciden con las esquinas de las baldosas.

\section{Discusión y conclusiones}

Desde el punto de vista matemático, las tareas propuestas resultaron propicias para reflexionar acerca de los elementos que determinan cada lugar geométrico abordado (circunferencia y bisectriz). En efecto, a pesar de que algunas situaciones no resultaron adecuadas para que emerja el concepto, la discusión posterior (durante la puesta en común de las Tareas 2 y 3) dio lugar a que, en la clase, se enuncien las condiciones que cumplen los puntos de la circunferencia (puntos de un plano que equidistan de otro punto fijo del mismo plano) y de la bisectriz (semirrecta interior de un ángulo cuyos puntos equidistan de los lados).

Asimismo, se dio la oportunidad de analizar en detalle (durante la discusión sobre la Actividad 4 del arco) los pasos a seguir para determinar puntos que pertenezcan a la bisectriz: "O sea, con la lana se podría haber tomado una distancia $x$, sin importar cuál sea, mientras siempre sea la misma, en realidad, la misma de este lado que de éste." Esta frase de Emilio (nombre ficticio) durante la puesta en común de la Tarea 2, explicita un modo de trazar puntos de la bisectriz. 
Por tanto, las consignas de las tareas se revelan adecuadas para que los futuros profesores doten de sentido (Gascón, 2004) las nociones trabajadas dado que se centró el análisis en los elementos identificatorios de cada lugar geométrico y en su trazado. Durante las Tareas 2 y 3 los sujetos reflexionaron sobre los conocimientos previos requeridos para la implementación de cada actividad en $5^{\circ}$ o $6^{\circ}$ de primaria y sobre posibles estrategias y dificultades de los niños. No obstante, la valoración de la posibilidad de que las situaciones resulten adecuadas para su implementación con niños de nivel primario queda fuera de los objetivos de este artículo.

Se observa, en coincidencia con lo postulado por Brinnitzer et al. (2018), que la posibilidad de aprender jugando se potencia si el docente recupera las estrategias y los conocimientos en acto construidos por los estudiantes. En efecto, la confusión entre la mediana y el segmento de bisectriz (en la Actividad del arco) pasó desapercibido para el GE y para la clase entera. Fue necesaria la intervención del docente con preguntas específicas: "¿se cercioraron de eso de que dividía al ángulo en dos partes iguales?, ¿de qué manera se cercioraron los que jugaron?" durante la puesta en común de la Tarea 2, para que los estudiantes tomen conciencia del error.

Es dispar el cumplimiento de los principios básicos que rigen la estructuración y aplicación de actividades lúdicas (Ortiz, 2014) en las situaciones diseñadas por los estudiantes. En efecto, todas carecen del principio de competencia. Éste quedaría reflejado mediante el establecimiento de objetivos que se deben alcanzar, dando así la oportunidad de definir ganadores o, si se trata de un juego individual, de identificar el momento en que el juego se completa. Por ejemplo, la representación del reloj (Actividad 1) no genera situación de rivalidad entre los participantes.

En general, las actividades propuestas tampoco constituyen desafios para los jugadores. Por ejemplo, en la Actividad 2 de la trayectoria del auto, los estudiantes son meros observadores de lo que un integrante del GD lleva a cabo. Es evidente que no se cumplen en esta situación y en la Actividad 5 de la búsqueda del tesoro los principios de participación, dinamismo y desempeño de roles.

Brinnitzer et al. (2018) mencionan la planificación de las secuencias y la selección de actividades por parte de los docentes como uno de los aspectos menos logrados. En particular, señalan que las situaciones diseñadas por los maestros no "demandaron diversos procesos cognitivos (comparar, definir, clasificar, resolver, calcular, etc.)". El estudio involucró docentes en ejercicio, en tanto que la investigación que se describe en el presente artículo se llevó a cabo con futuros docentes, por lo que resulta razonable esperar que estos 
últimos presenten dificultades a la hora de diseñar actividades de aprendizaje mediadas por el juego.

Estas observaciones dan cuenta de la necesidad de destinar en la formación inicial de docentes de primaria, espacios para abordar la noción de juego didáctico y sus características. Si bien en la primera clase se desarrolló un juego para construir el concepto de mediatriz, que cumplía con los principios básicos ya mencionados, la actividad resultó insuficiente para que los estudiantes reflexionen sobre ellos y los transfieran a la planificación de sus propias situaciones diseñadas.

Cabe señalar que los estudiantes, en general, lograron identificar las limitaciones señaladas en los párrafos precedentes. Esto se desprende de los siguientes comentarios, obtenidos a partir de una encuesta implementada con el fin de recuperar sus reflexiones sobre el trabajo realizado:

Fortalezas:

- Es una mirada distinta para un aprendizaje significativo de saberes nuevos.

- La experiencia realizada nos permitió comprender cómo las actividades lúdicas favorecen la adquisición del conocimiento y despiertan el interés en los alumnos. Además, pudimos identificar qué cuestiones debemos tener en cuenta a la hora de planificar dichas actividades.

- Al enseñar geometría a través de un juego luego es más fácil relacionarla o encontrarla en la realidad.

Debilidades:

- Nuestra propuesta didáctica resultó ser errónea, dado que en la misma, no emergian los conceptos que debiamos enseñar. No obstante, esto nos sirvió como aprendizaje para futuras experiencias.

- Confusión al momento de plantear el juego ya que propusimos la comparación entre círculo y circunferencia y no emergió el concepto de circunferencia.

Sugerencias

- Ser más detallista en cada paso a realizar para asegurar que los conceptos sean bien adquiridos.

- Como grupo, consideramos importante una enseñanza previa acerca de cómo pensar, crear y planificar un juego didáctico, para asegurarnos que surjan los conceptos correctamente.

- Tener claros los conceptos que se van a trabajar.

Los comentarios de los estudiantes dan cuenta de la realización de un trabajo de metacognición, que les permitió identificar las 
cuestiones a ajustar y a tener en cuenta para experiencias similares. Desde el punto de vista de la formación de docentes para la educación primaria, se reconoce que la complejidad de las prácticas requiere de la "reflexión y la metacognición como dispositivo de comprensión y análisis" de las mismas (Gobierno de Santa Fe. Ministerio de Educación, 2009, p.49). En esta propuesta, se ha intentado otorgarle un lugar especial a la reflexión sobre lo realizado, tanto en la encuesta final como en las Tareas 2 y 3, que tenian como fin revisar las actividades implementadas.

El estudio ha puesto de manifiesto que la consigna de diseñar juegos didácticos en una clase de futuros docentes de primaria permite identificar con claridad los rasgos que determinan a las figuras geométricas, cuando se destinan espacios para intercambiar y discutir las producciones. Además, ha evidenciado el rol central del docente para plantear interrogantes que permitan explorar nuevos conocimientos.

Finalmente, los resultados muestran algunas dificultades que surgen durante el diseño de situaciones lúdicas, lo cual abre un nuevo interrogante de investigación: ¿qué tipo de experiencias resultan valiosas, durante la formación inicial del profesor de primaria, para promover la identificación y el diseño de juegos didácticos que permitan fortalecer la formación matemática de los estudiantes?

\section{Referencias}

Bosch, M., García, F.J., Gascón, J. y Ruiz Higueras, L., (2006). La modelización matemática y el problema de la articulación de la matemática escolar. Una propuesta desde la teoría antropológica de lo didáctico. Educación Matemática, 18(2), 37-74. http: / / funes.uniandes.edu.co/13119/1/Bosch2006La.pdf

Bosch, M. y Gascón, J. (2009). Aportaciones de la Teoría Antropológica de lo Didáctico a la formación del profesorado de matemáticas de secundaria. En M.J. González, M.T. González \& J. Murillo (Eds.), Investigación en Educación Matemática XIII (pp. 89-113). Santander:

SEIEM. https: / / documat.unirioja.es / servlet/articulo?codigo $=3628647$

Bernabeu, M., Llinares, S. y Moreno, M. (2017). Características de la comprensión de figuras geométricas en estudiantes de 6 a 12 años. En J.M. Muñoz-Escolano, A. Arnal-Bailera, P. BeltránPellicer, M.L. Callejo y J. Carrillo (Eds.), Investigación en 
Educación Matemática XXI (pp. 157-166). Zaragoza: SEIEM.

Bernabeu, M., Moreno, M. y Llinares, S. (2018). Cómo estudiantes para maestro/a anticipan posibles respuestas de niños/as en actividades de reconocimiento de figuras geométrica. En R. RoigVila (Ed.), El compromiso académico y social a través de la investigación e innovación educativas en la Enseñanza Superior (pp.59-68). Barcelona: Ediciones OCTAEDRO, S.L.

Bravo, C., Márquez, H., y Villarroel, F. (2013). Los juegos como estrategia metodológica en la enseñanza de la geometría, en estudiantes de séptimo grado de educación básica. Revista Digital: Matemática, Educación E Internet, 13(1). https://doi.org/10.18845/rdmei.v13i1.1624

Brinnitzer, E.V., Collado, M.E., Gallego, M.F. y Pérez, S.G. (2018). E1 juego y el aprendizaje de la divisibilidad en el Nivel Primario . En L. Vesub (Coord.), La investigación en los Institutos de Formación Docente. Volumen 1. Prácticas docentes y de enseñanza (pp.302326). Buenos Aires: Ministerio de Educación, Cultura, Ciencia y Tecnologia.

https://www.educ.ar/sitios/educar/resources/150626/practica s-docentes-y-de-enseanza/download

Chevallard, Y. (2013). La matemática en la escuela. Por una revolución epistemológica y didáctica. Buenos Aires: libros del Zorzal.

Collado, M., Fernández Panizza, G., Gallego, M.F., Pacheco, S., Pérez, S., Santamaría, F. y Simari, C. (2011). El juego en la enseñanza de la matemática. Un estudio sobre las concepciones de estudiantes y docentes acerca del juego en el aprendizaje y la enseñanza de la matemática. Informe final de investigación. http: / /gpdmatematica.org.ar/wpcontent/uploads/2015/08/El_juego_bariloche.pdf

Franco Mariscal, A.J. y Simeoli Sánchez, P.S. (2019). Un enfoque basado en juegos educativos para aprender geometría en educación primaria: Estudio preliminar. Educação e Pesquisa, 45, 1-24. https://dx.doi.org/10.1590/s16784634201945184114

Gascón, J. (2004). Efectos del autismo temático sobre el estudio de la Geometría en Secundaria II. La clasificación de los cuadriláteros convexos. SUMA, 45 , 41-52. 
https://revistasuma.es/IMG/pdf/45/041-052.pdf

Gobierno de Santa Fe. Ministerio de Educación (2009). Profesorado de Educación Primaria. Diseño Curricular para la Formación Docente. https://www.santafe.gov.ar/index.php/educacion/content/down load/122509/606630/file/528-09\%20Primario.pdf

Kiener, F. y Milani, C. (2019). Estudio de tareas lúdicas para abordar nociones geométricas con futuros docentes del nivel primario. En J. Aguirre, L. Proasi y C. De Laurentis (Comps.), Congreso Latinoamericano "Prácticas, problemáticas y desafios contemporáneos de la Universidad y del Nivel Superior". ACTA DE COMUNICACIONES Eje 12. https://drive.google.com/file/d/1IGqAUlICXDzEZOVaxqfNj734w316T8P/view

Ministerio de Educación Provincia de Santa FE (2016). Núcleos Interdisciplinarios de Contenidos. Recursos para su enseñanza. https:// plataformaeducativa.santafe.gov.ar/moodle/pluginfile.ph p/327637/mod_resource/content/1/NIC\%202\%20recursos.pdf

Muñiz-Rodríguez, L., Alonso, P. y Rodríguez-Muñiz, L.J. (2014). El uso de los juegos como recurso didáctico para la enseñanza y el aprendizaje de las Matemáticas: estudio de una experiencia innovadora. Unión, 39, 19-33. http://www.fisem.org/www/union/revistas/2014/39/archivo6.p df

Ortiz Ocaña, A. (2014). Educación Infantil. ¿Cómo estimular y evaluar el desarrollo cognitivo del niño y afectivo de los niños y niñas desde el aula de clase? Bogotá: Ediciones de la U.

Rodríguez Rava, B. (2015). Algunas reflexiones sobre la enseñanza de la Geometría en la escuela primaria. Quehacer educativo, XXV (133), 12-19.

https://uruguayeduca.anep.edu.uy/sites/default/files/2019-

02/Algunas reflexiones sobre la enseñanza del a geometría en la escuela primaria.pdf

Ruiz Olarria, A., Sierra Delgado, T.A., Bosch Casabó, M. y Gascón Pérez, J. (2014). Las Matemáticas para la Enseñanza en una Formación del Profesorado Basada en el Estudio de Cuestiones. Bolema, 28(48), 319-340. http://www.scielo.br/pdf/bolema/v28n48/16.pdf 
Sadovsky, P. (coord.) (2010). La enseñanza de la matemática en la formación docente para la escuela primaria. Buenos Aires: Ministerio de Educación de la Nación. https://cedoc.infd.edu.ar/upload/Investigacion_nacional_SADO VSKY_.PDF

Sierra Delgado, T.A., Bosch Casabó, M. y Gascón Pérez, J. (2012). La formación matemático-didáctica del maestro de Educación Infantil: el caso de "cómo enseñar a contar". Revista de Educación, 357 , 231-256. https://pdfs.semanticscholar.org/5d2e/f2866c90df41704e1f9d3 920508fd73e952a.pdf 